\title{
TORIC IDEALS OF FINITE GRAPHS AND ADJACENT 2-MINORS
}

\author{
HIDEFUMI OHSUGI AND TAKAYUKI HIBI
}

\begin{abstract}
We study the problem when an ideal generated by adjacent 2-minors is the toric ideal of a finite graph.
\end{abstract}

Let $X=\left(x_{i j}\right)_{\substack{i=1, \ldots, m \\ j=1, \ldots, n}}$ be a matrix of $m n$ indeterminates, and let $A=K\left[\left\{x_{i j}\right\}_{\substack{i=1, \ldots, m \\ j=1, \ldots, n}}\right]$ be the polynomial ring in $m n$ variables over a field $K$. Given $1 \leq a_{1}<a_{2} \leq m$ and $1 \leq b_{1}<b_{2} \leq n$, the symbol $\left[a_{1}, a_{2} \mid b_{1}, b_{2}\right]$ denotes the 2-minor $x_{a_{1} b_{1}} x_{a_{2} b_{2}}-x_{a_{1} b_{2}} x_{a_{2} b_{1}}$ of $X$. In particular $\left[a_{1}, a_{2} \mid b_{1}, b_{2}\right]$ is a binomial of $A$. A 2-minor $\left[a_{1}, a_{2} \mid b_{1}, b_{2}\right]$ of $X$ is adjacent ([4]) if $a_{2}=a_{1}+1$ and $b_{2}=b_{1}+1$. Following [2], we say that a set $\mathcal{M}$ of adjacent 2-minors of $X$ is of chessboard type if the following conditions are satisfied:

- if $[a, a+1 \mid b, b+1]$ and $\left[a, a+1 \mid b^{\prime}, b^{\prime}+1\right]$ with $b<b^{\prime}$ belong to $\mathcal{M}$, then $b+1<b^{\prime}$

- if $[a, a+1 \mid b, b+1]$ and $\left[a^{\prime}, a^{\prime}+1 \mid b, b+1\right]$ with $a<a^{\prime}$ belong to $\mathcal{M}$, then $a+1<a^{\prime}$.

Given a set $\mathcal{M}$ of adjacent 2-minors of $X$ of chessboard type, we introduce the finite graph $\Gamma_{\mathcal{M}}$ on the vertex set $\mathcal{M}$, whose edges are $\left\{[a, a+1 \mid b, b+1],\left[a^{\prime}, a^{\prime}+1 \mid b^{\prime}, b^{\prime}+1\right]\right\}$ such that

- $[a, a+1 \mid b, b+1] \neq\left[a^{\prime}, a^{\prime}+1 \mid b^{\prime}, b^{\prime}+1\right]$,

- $\{a, a+1\} \cap\left\{a^{\prime}, a^{\prime}+1\right\} \neq \emptyset$,

- $\{b, b+1\} \cap\left\{b^{\prime}, b^{\prime}+1\right\} \neq \emptyset$.

For example, if $\mathcal{M}=\{[1,2 \mid 2,3],[2,3 \mid 3,4],[3,4 \mid 2,3],[2,3 \mid 1,2]\}$, then $\Gamma_{\mathcal{M}}$ is a cycle of length 4 . The ideal $I_{\mathcal{M}}$ is generated by $x_{12} x_{23}-x_{13} x_{22}, x_{23} x_{34}-x_{24} x_{33}, x_{32} x_{43}-x_{33} x_{42}$ and $x_{21} x_{32}-x_{22} x_{31}$. The binomial $x_{32}\left(x_{13} x_{21} x_{34} x_{42}-x_{12} x_{24} x_{31} x_{43}\right)$ belongs to $I_{\mathcal{M}}$ but neither $x_{32}$ nor $x_{13} x_{21} x_{34} x_{42}-x_{12} x_{24} x_{31} x_{43}$ belongs to $I_{\mathcal{M}}$. Thus $I_{\mathcal{M}}$ is not prime.

A fundamental fact regarding ideals generated by adjacent 2-minors is

Lemma 1 ([2]). Let $\mathcal{M}$ be a set of adjacent 2 -minors of $X$, and let $I_{\mathcal{M}}$ be the ideal of $A$ generated by all 2-minors belonging to $\mathcal{M}$. Then, $I_{\mathcal{M}}$ is a prime ideal if and only if $\mathcal{M}$ is of chessboard type, and $\Gamma_{\mathcal{M}}$ possesses no cycle of length 4.

A finite graph $G$ is said to be simple if $G$ has no loop and no multiple edge. Let $G$ be a finite simple graph on the vertex set $[d]=\{1, \ldots, d\}$, and let $E(G)=$

2010 Mathematics Subject Classification: Primary 13F20.

Keywords: Toric ideals, Ideals of 2-adjacent minors, finite graphs.

This research was supported by JST CREST . 
$\left\{e_{1}, \ldots, e_{n}\right\}$ be its set of edges. Let $K[\mathbf{t}]=K\left[t_{1}, \ldots, t_{d}\right]$ denote the polynomial ring in $d$ variables over $K$, and let $K[G]$ denote the subring of $K[\mathbf{t}]$ generated by the squarefree quadratic monomials $\mathbf{t}^{e}=t_{i} t_{j}$ with $e=\{i, j\} \in E(G)$. The semigroup ring $K[G]$ is called the edge ring of $G$. Let $K[\mathbf{y}]=K\left[y_{1}, \ldots, y_{n}\right]$ denote the polynomial ring in $n$ variables over $K$. The kernel $I_{G}$ of the surjective homomorphism $\pi: K[\mathbf{y}] \rightarrow K[G]$ defined by setting $\pi\left(y_{i}\right)=\mathbf{t}^{e_{i}}$ for $i=1, \ldots, n$ is called the toric ideal of $G$. Clearly, $I_{G}$ is a prime ideal. It is known that $I_{G}$ is generated by the binomials corresponding to even closed walks of $G$. See [7], [6, Chapter 9] and [5, Lemma 1.1] for details.

Example 2. Let $G$ be a complete bipartite graph with the edge set $E(G)=\{\{i, p+$ $j\} \mid 1 \leq i \leq p, \quad 1 \leq j \leq q\}$. Let $X=\left(x_{i j}\right)_{\substack{i=1, \ldots, p \\ j=1, \ldots, q}}$ be a matrix of $p q$ indeterminates and $K[\mathbf{x}]=K\left[\left\{x_{i j}\right\}_{\substack{i=1, \ldots, p \\ j=1, \ldots, q}}\right.$. Then, $I_{G}$ is the kernel of the surjective homomorphism $\pi: K[\mathbf{x}] \rightarrow K[G]$ defined by setting $\pi\left(x_{i j}\right)=t_{i} t_{p+j}$ for $1 \leq i \leq p, 1 \leq j \leq q$. It is known [6, Proposition 5.4] that $I_{G}$ is generated by the set of all 2-minors of $X$. Note that each 2-minor $x_{i j} x_{i^{\prime} j^{\prime}}-x_{i j^{\prime}} x_{i^{\prime} j}$ corresponds to the cycle $\{\{i, p+j\},\{p+$ $\left.\left.j, i^{\prime}\right\},\left\{i^{\prime}, p+j^{\prime}\right\},\left\{p+j^{\prime}, i\right\}\right\}$ of $G$.

In general, a toric ideal is the defining ideal of a homogeneous semigroup ring. We refer the reader to [6] for detailed information on toric ideals. It is known [1] that a binomial ideal $I$, i.e., an ideal generated by binomials, is a prime ideal if and only if $I$ is a toric ideal. An interesting research problem on toric ideals is to determine when a binomial ideal is the toric ideal of a finite graph.

Example 3. The ideal $I=\left\langle x_{1} x_{2}-x_{3} x_{4}, x_{1} x_{2}-x_{5} x_{6}, x_{1} x_{2}-x_{7} x_{8}\right\rangle$ is the toric ideal of the semigroup ring $K\left[t_{1} t_{5}, t_{2} t_{3} t_{4} t_{5}, t_{1} t_{2} t_{5}, t_{3} t_{4} t_{5}, t_{2} t_{3} t_{5}, t_{1} t_{4} t_{5}, t_{1} t_{3} t_{5}, t_{2} t_{4} t_{5}\right]$. If there exists a graph $G$ such that $I=I_{G}$, then three quadratic binomials correspond to cycles of length 4 . However, this is impossible since these three cycles must have common two edges $e_{1}$ and $e_{2}$ such that $e_{1} \cap e_{2}=\emptyset$. Thus, $I$ cannot be the toric ideal of a finite graph. This observation implies that the toric ideal of a finite distributive lattice $\mathcal{L}$ (see [3]) is the toric ideal of a finite graph if and only if $\mathcal{L}$ is planar. In fact, if $\mathcal{L}$ is planar, then it is easy to see that the toric ideal of $\mathcal{L}$ is the toric ideal of a bipartite graph. If $\mathcal{L}$ is not planar, then $\mathcal{L}$ contains a sublattice that is isomorphic to the Boolean lattice $B_{3}$ of rank 3. Since the toric ideal of $B_{3}$ has three binomials above, the toric ideal of $\mathcal{L}$ cannot be the toric ideal of a finite graph.

Let $\mathcal{M}$ be a set of adjacent 2-minors. Now, we determine when a binomial ideal $I_{\mathcal{M}}$ generated by $\mathcal{M}$ is the toric ideal $I_{G}$ of a finite graph $G$. Since $I_{G}$ is a prime ideal, according to Lemma 1, if there exists a finite graph $G$ with $I_{\mathcal{M}}=I_{G}$, then $\mathcal{M}$ must be of chessboard type and $\Gamma_{\mathcal{M}}$ possesses no cycle of length 4 .

Theorem 4. Let $\mathcal{M}$ be a set of adjacent 2-minors. Then, there exists a finite graph $G$ such that $I_{\mathcal{M}}=I_{G}$ if and only if $\mathcal{M}$ is of chessboard type, $\Gamma_{\mathcal{M}}$ possesses no cycle of length 4 , and each connected component of $\Gamma_{\mathcal{M}}$ possesses at most one cycle. 
Proof. We may assume that $\mathcal{M}$ is of chessboard type and $\Gamma_{\mathcal{M}}$ possesses no cycle of length 4. Let $\mathcal{M}=\mathcal{M}_{1} \cup \cdots \cup \mathcal{M}_{s}$, where $\Gamma_{\mathcal{M}_{1}}, \ldots, \Gamma_{\mathcal{M}_{s}}$ is the set of connected components of $\Gamma_{\mathcal{M}}$. If $i \neq j$, then $f \in \mathcal{M}_{i}$ and $g \in \mathcal{M}_{j}$ have no common variable. Hence, there exists a finite graph $G$ such that $I_{\mathcal{M}}=I_{G}$ if and only if for each $1 \leq i \leq s$, there exists a finite graph $G_{i}$ such that $I_{\mathcal{M}_{i}}=I_{G_{i}}$. Thus, we may assume that $\Gamma_{\mathcal{M}}$ is connected. Let $p$ be the number of vertices of $\Gamma_{\mathcal{M}}$, and let $q$ be the number of edges of $\Gamma_{\mathcal{M}}$. Since $\Gamma_{\mathcal{M}}$ is connected, we have $p \leq q+1$.

[Only if] Suppose that there exists a finite graph $G$ with $I_{\mathcal{M}}=I_{G}$. From [2, Theorem 2.3], the codimension of $I_{\mathcal{M}}$ is equal to $p$. Let $d$ be the number of vertices of $G$, and let $n$ be the number of edges of $G$. Then, we have $d \leq 4 p-2 q$ and $n=4 p-q$. The height of $I_{G}$ is given in [7]. If $G$ is bipartite, then the codimension of $I_{G}$ satisfies $p \geq n-d+1 \geq(4 p-q)-(4 p-2 q)+1=q+1$. Hence, we have $p=q+1$ and $\Gamma_{\mathcal{M}}$ is a tree. On the other hand, if $G$ is not bipartite, then the codimension of $I_{G}$ satisfies $p \geq n-d \geq(4 p-q)-(4 p-2 q)=q$. Hence, we have $p \in\{q, q+1\}$ and $\Gamma_{\mathcal{M}}$ has at most one cycle.

[If] Suppose that $\Gamma_{\mathcal{M}}$ has at most one cycle. Then, we have $p \in\{q, q+1\}$.

Case 1. $p=q+1$, i.e., $\Gamma_{\mathcal{M}}$ is a tree.

Through induction on $p$, we will show that there exists a connected bipartite graph $G$ such that $I_{\mathcal{M}}=I_{G}$. If $p=1$, then $I_{\mathcal{M}}=I_{G}$ where $G$ is a cycle of length 4. Let $k>1$, and suppose that the assertion holds for $p=k-1$. Suppose that $\Gamma_{\mathcal{M}}$ has $k$ vertices. Since $\Gamma_{\mathcal{M}}$ is a tree, $\Gamma_{\mathcal{M}}$ has a vertex $v=[a, a+1 \mid b, b+1]$ of degree 1. Let $\mathcal{M}^{\prime}=\mathcal{M} \backslash\{v\}$. Since $\Gamma_{\mathcal{M}^{\prime}}$ is a tree, there exists a connected bipartite graph $G^{\prime}$ such that $I_{\mathcal{M}^{\prime}}=I_{G^{\prime}}$ by the hypothesis of induction. From [5, Theorem 1.2], since $I_{G^{\prime}}$ is generated by quadratic binomials, any cycle of $G^{\prime}$ of length $\geq 6$ has a chord. Let $v^{\prime}=\left[a^{\prime}, a^{\prime}+1 \mid b^{\prime}, b^{\prime}+1\right]$ denote the vertex of $\Gamma_{\mathcal{M}}$ that is incident with $v$. Let $e=\{i, j\}$ be the edge of $G^{\prime}$ corresponding to the common variable of $v$ and $v^{\prime}$. Let $\{1,2, \ldots, d\}$ be the vertex set of $G^{\prime}$. We now define the connected bipartite graph $G$ on the vertex set $\{1,2, \ldots, d, d+1, d+2\}$ with the edge set $E\left(G^{\prime}\right) \cup\{\{i, d+1\},\{d+1, d+2\},\{d+2, j\}\}$. Then, any cycle of $G$ of length $\geq 6$ has a chord, and hence, $I_{G}$ is generated by quadratic binomials. Thus, $I_{G}$ is generated by the quadratic binomials of $I_{G^{\prime}}$ together with $v$ corresponding to the cycle $\{\{i, d+1\},\{d+1, d+2\},\{d+2, j\},\{j, i\}\}$. Therefore, $I_{\mathcal{M}}=I_{G}$.

Case 2. $p=q$, i.e., $\Gamma_{\mathcal{M}}$ has exactly one cycle.

Then, we have $p \geq 8$. Through induction on $p$, we will show that there exists a graph $G$ such that $I_{\mathcal{M}}=I_{G}$. If $p=8$, then $\Gamma_{\mathcal{M}}$ is a cycle of length 8 . Then, $I_{\mathcal{M}}=I_{G}$ where $G$ is the graph shown in Figure 1 .

Let $k>8$ and suppose that the assertion holds for $p=k-1$. Suppose that $\Gamma_{\mathcal{M}}$ has $k$ vertices. If $\Gamma_{\mathcal{M}}$ has a vertex $v=[a, a+1 \mid b, b+1]$ of degree 1 , then $\Gamma_{\mathcal{M}^{\prime}}$ where $\mathcal{M}^{\prime}=\mathcal{M} \backslash\{v\}$ has exactly one cycle, and hence, there exists a graph $G^{\prime}$ such that $I_{\mathcal{M}^{\prime}}=I_{G^{\prime}}$ by the hypothesis of induction. Let $v^{\prime}=\left[a^{\prime}, a^{\prime}+1 \mid b^{\prime}, b^{\prime}+1\right]$ denote the 


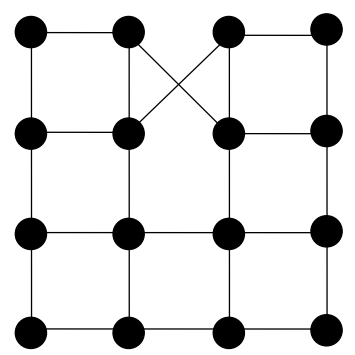

Figure 1 . Graph for $\mathcal{M}$ such that $\Gamma_{\mathcal{M}}$ is a cycle of length 8 .

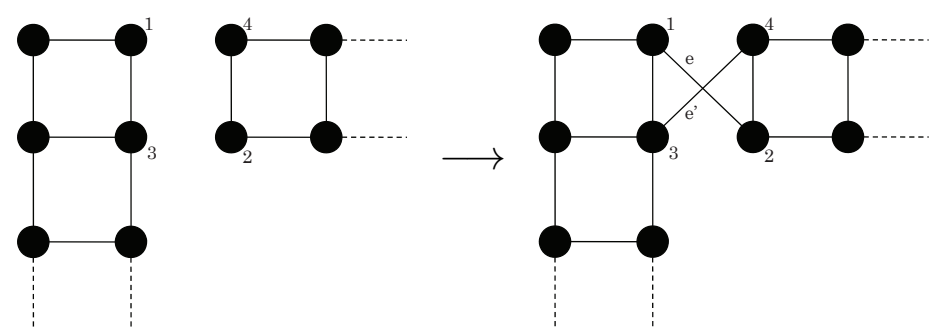

FiguRE 2. New graph $G$ arising from $G^{\prime}$.

vertex of $\Gamma_{\mathcal{M}}$ that is incident with $v$. Let $e=\{i, j\}$ be the edge of $G^{\prime}$ corresponding to the common variable of $v$ and $v^{\prime}$. Suppose that the vertex set of $G^{\prime}$ is $\{1,2, \ldots, d\}$. We now define the graph $G$ on the vertex set $\{1,2, \ldots, d, d+1, d+2\}$ with the edge set $E\left(G^{\prime}\right) \cup\{\{i, d+1\},\{d+1, d+2\},\{d+2, j\}\}$. Since $G^{\prime}$ satisfies the conditions in [5. Theorem 1.2], it follows that $G$ satisfies the conditions in [5, Theorem 1.2]. Thus, $I_{G}$ is generated by the quadratic binomials of $I_{G^{\prime}}$ together with $v$ corresponding to the cycle $\{\{i, d+1\},\{d+1, d+2\},\{d+2, j\},\{j, i\}\}$. Therefore, $I_{\mathcal{M}}=I_{G}$.

Suppose that $\Gamma_{\mathcal{M}}$ has no vertex of degree 1 . Then, $\Gamma_{\mathcal{M}}$ is a cycle of length $k$. A 2-minor $a d-b c \in \mathcal{M}$ is called free if one of the following holds:

- Neither $a$ nor $d$ appears in other 2-minors of $\mathcal{M}$,

- Neither $b$ nor $c$ appears in other 2-minors of $\mathcal{M}$.

From [2, Lemma 1.6], $\mathcal{M}$ has at least two free 2-minors. Let $v=[a, a+1 \mid b, b+1]$ be a free 2-minor of $\mathcal{M}$. We may assume that neither $x_{a, b}$ nor $x_{a+1, b+1}$ appears in other 2-minors of $\mathcal{M}$. Since $\Gamma_{\mathcal{M}}$ is a cycle, $x_{a+1, b}$ appears in exactly two 2-minors of $\mathcal{M}$ and $x_{a, b+1}$ appears in exactly two 2-minors of $\mathcal{M}$. Let $\mathcal{M}^{\prime}=\mathcal{M} \backslash\{v\}$. Since $\Gamma_{\mathcal{M}^{\prime}}$ is a tree, there exists a connected bipartite graph $G^{\prime}$ such that $I_{\mathcal{M}^{\prime}}=I_{G^{\prime}}$ by the argument in Case 1. Suppose that the edge $\{1,3\}$ corresponds to the variable $x_{a+1, b}$ and the edge $\{2,4\}$ corresponds to the variable $x_{a, b+1}$. We now define the graph $G$ as shown in Figure 2, where vertices 1 and 2 belong to the same part of the bipartite graph $G^{\prime}$. Note that $G$ is not bipartite. Let $e=\{1,2\}$ and $e^{\prime}=\{3,4\}$. Since $G^{\prime}$ is a bipartite graph, it follows that 
(a) If either $e$ or $e^{\prime}$ is an edge of an even cycle $C$ of $G$, then $\left\{e, e^{\prime}\right\} \subset E(C)$.

(b) If $C^{\prime}$ is an odd cycle of $G$, then $\left\{e, e^{\prime}\right\} \cap E\left(C^{\prime}\right) \neq \emptyset$.

Let $I$ denote the ideal generated by all quadratic binomials in $I_{G}$. Since each quadratic binomial in $I_{G}$ corresponds to a cycle of $G$ of length 4 , it follows that $I_{\mathcal{M}}=I$. Thus, it is sufficient to show that $I_{G}=I$, i.e., $I_{G}$ is generated by quadratic binomials. From [5, Theorem 1.2], since $G^{\prime}$ is bipartite and since $I_{G^{\prime}}$ is generated by quadratic binomials, all cycles of $G^{\prime}$ of length $\geq 6$ have a chord.

Let $C$ be an even cycle of $G$ of length $\geq 6$. If $E(C) \cap\left\{e, e^{\prime}\right\}=\emptyset$, then $C$ has an even-chord since all cycles of the bipartite graph $G^{\prime}$ of length $\geq 6$ have a chord. Suppose that $\left\{e, e^{\prime}\right\} \subset E(C)$ holds. Then, either $\{1,3\}$ or $\{2,4\}$ is a chord of $C$. Moreover, such a chord is an even-chord of $C$ from (b) above.

Let $C$ and $C^{\prime}$ be odd cycles of $G$ having exactly one common vertex. From (b) above, we may assume that $e \in E(C) \backslash E\left(C^{\prime}\right)$ and $e^{\prime} \in E\left(C^{\prime}\right) \backslash E(C)$. If $\{1,3\}$ does not belong to $E(C) \cup E\left(C^{\prime}\right)$, then $\{1,3\}$ satisfies the condition in [5, Theorem 1.2 (ii)]. If $\{1,3\}$ belongs to $E(C) \cup E\left(C^{\prime}\right)$, then $\{2,4\} \notin E(C) \cup E\left(C^{\prime}\right)$ since $C$ and $C^{\prime}$ have exactly one common vertex. Hence, $\{2,4\}$ satisfies the condition in $[5$, Theorem 1.2 (ii)].

Let $C$ and $C^{\prime}$ be odd cycles of $G$ having no common vertex. Then, neither $\{1,3\}$ nor $\{2,4\}$ belong to $E(C) \cup E\left(C^{\prime}\right)$. Hence, $\{1,3\}$ and $\{2,4\}$ satisfy the condition in [5, Theorem 1.2 (iii)].

Thus, from [5, Theorem 1.2], $I_{G}$ is generated by quadratic binomials. Therefore, $I_{G}=I_{\mathcal{M}}$ as desired.

\section{REFERENCES}

[1] D. Eisenbud and B. Sturmfels, Binomial ideals, Duke Math. J. 84 (1996), 1-45.

[2] J. Herzog and T. Hibi, Ideals generated by adjacent 2-minors, J. Comm. Algebra, to appear.

[3] T. Hibi, Distributive lattices, affine semigroup rings and algebras with straightening laws, in "Commutative Algebra and Combinatorics" (M. Nagata and H. Matsumura, Eds.), Adv. Stud. Pure Math., Volume 11, North-Holland, Amsterdam, 1987, pp. 93-109.

[4] S. Hoşten and S. Sullivant, Ideals of adjacent minors, J. Algebra 277 (2004), 615-642.

[5] H. Ohsugi and T. Hibi, Toric ideals generated by quadratic binomials, J. Algebra 218 (1999), $509-527$.

[6] B. Sturmfels, "Gröbner bases and convex polytopes," Amer. Math. Soc., Providence, RI, 1996.

[7] R. Villarreal, Rees algebras of edge ideals, Comm. Algebra 23 (1995), 3513-3524.

Hidefumi Ohsugi, Department of Mathematics, College of Science, Rikkyo UniVERSITY, TOSHIMA-KU, TOKYO 171-8501, JAPAN

E-mail address: ohsugi@rikkyo.ac.jp

Takayuki Hibi, Department of Pure and Applied Mathematics, Graduate School of Information Science and Technology, Osaka University, Toyonaka, Osaka 5600043, JAPAN

E-mail address: hibi@math.sci.osaka-u.ac.jp 